\title{
XII. Abstracts of a series of papers lately read before the Royal Society, \&c. on the magnetism developed by rotation
}

\author{
Messr. Barlow, Messr. Christie, Messr. Babbage, Messr. Herschel \& Messr. \\ Marsh
}

To cite this article: Messr. Barlow, Messr. Christie, Messr. Babbage, Messr. Herschel \& Messr. Marsh (1825) XII. Abstracts of a series of papers lately read before the Royal Society, \&c. on the magnetism developed by rotation, Philosophical Magazine Series 1, 66:328, 93-102, DOI: 10.1080/14786442508673929

To link to this article: http://dx.doi.org/10.1080/14786442508673929

曲 Published online: 10 Aug 2009.

Submit your article to this journal $₫$

山 Article views: 3

Q View related articles $\longleftarrow$ 
Mr. Barlow on the Magnetism developed by Rotation. 93

wards or downwards when they have been accidentally excited. The extreme limits between which every atmosphere possessing these properties is contained are, on the one hand, the atmosphere imagined by Dalton, in which every mass of air retains the whole of its absolute heat without increase or diminution; and, on the other, the atmosphere of equable temperature, in which a mass of air has the whole heat absorbed by rarefaction restored to it. In these extreme atmospheres the only action which the aggregate exerts upon its parts is pressure; and every partial mass, having the sources of temperature entirely within itself, is indifferent to motion or rest, since in either case the elasticity will equally balance the external pressure. In any of the intermediate atmospheres, when a mass of air shifts its place, its elasticity varies both on account of the change of volume and the transference of heat; and as these two causes produce effects contrary to one another, there is introduced a principle of stability tending to destroy vertical motions upwards or downwards. By the foregoing reasoning the investigation of the true atmosphere is at least brought within certain limits. In proceeding further, we must have recourse to other phænomena, in order to particularize the individual case that alone agrees with nature in all its properties. But we may here observe that the principle of stability, which is evanescent in both the extreme cases, must first increase and then decrease in passing from one to the other. At some intermediate point it will therefore operate with a maximum effect; and it is not unreasonable to conjecture that this particular case, merely because it is single, will be found to agree with the atmosphere of nature. This, however, is a point to be inquired into, not one conclusively established.

Aug. 2, 1825.

[To be continued.]

JAMES Ivory.

XII. Abstracts of a Series of Papers lately read before the Royal Society, \&c. on the Magnetism developed by Rotation. By Messrs. Barlow, Christie, Babbage, Herschel, and MarSH *.

On the Magnetism imparted to Iron Bodies by Rotation. By Peter Barlow, Esq. F.R.S.

THE author's attention having been recalled to the consideration of the effects of rotation in altering the magnetic influence of iron, in the course of speculations on the cause of the

* The great importance of the experiments and investigations detailed 
the rotation of the earth's magnetic poles, and knowing at the same time that Mr. Christie had found a permanent change in the magnetic state of an iron plate by a mere change of position on its axis, it seemed to him highly probable that this change, due only to a simple inversion, would be increased by rapid rotation. On trial, however, it was found that the effect produced was merely temporary. The experiments at first were made with a thirteen-inch mortar-shell fixed to the mandrel of a powerful turning lathe, worked by a steam-engine in the royal arsenal at Woolwich.

This being made to revolve at the rate of 640 turns per minute, the needle was deflected out several degrees, and there remained stationary during the motion of the ball, but returned immediately to its original position on ceasing the rotation. On inverting the motion of the shell, an equal and contrary deflection took place.

As the law of the phænomena was not evident with this disposition of the apparatus, and the shell was found too heavy for perfect safety, a Shrapnell shell of eight inch diameter was mounted in a proper apparatus (described in the paper), and a number of experiments made, the law of which, however, still seemed anomalous, till the idea oecurred of neutralizing the earth's action on the needle; when the anomalies disappeared, and the general law of the effect was placed in evidence. The needle being made a tangent to the ball, if the ball was made to revolve torwards the needle (whatever was the direction of the axis of rotation), the north end of the latter was attracted, and if the contrary way, repelled. In the two extremities of the axis there was found no effect; while in two opposite points at right angles to the axis, the effect was a maximam, and the direction of the needle was to the centre of the ball.

The author then proceeded to show how the results, which before appeared anomalous, agree with this general view, and closed his communication with some theoretical views of their general bearing on the subjects of the earth's magnetism, which he thought there were strong reasons for believing to be of the induced kind; and although it appeared to him doubtful whether the anomalies observed in the variation of the needle on the earth's surface can ultimately be referred to this cause, yet he observed that one condition essential to the production

in these papers has determined us to give in a connected form the official abstracts of them, 'as read before the Royal Soeiety, from the Quarterly Journal of Science. We have added, to complete the series, Mr. Marsh's account of Mr. Barlow's repetition of M. Arago's late experiments, from the Edinburgh Philosophical Journal. 
of these phænomena holds good in the case of the earth, viz. the non-coincidence of its polarized axis with that of its diurnal rotation.

On the Alteration in the Magnetism of an Iron Plate, occasioned by a Rotation on its Axis. By S. H. Christie, Esq.

The effects observed and described in this paper, although minute in themselves, appeared, in the author's opinion, to point out a species of magnetic action not hitherto described. It has long been well known that striking, twisting, or filing iron, in different directions, with regard to the magnetic axis, materially influences its polarity, but it does not appear to have been remarked that the simple rotation of iron in different directions has any such influence. This, however, the author has ascertained to be the case, and that the laws which govern this peculiar action are so regular, that there can remain no doubt of a corresponding regularity in their causes.

The attention of the author was first drawn to these phænomena by some apparent anomalies in the magnetic action of an iron plate on the compass, observed in the course of a different investigation. In order to avoid or allow for the disturbing influence of partial magnetism in the iron, it became necessary to attend minutely to the position of certain points in its circumference, which corresponded to the maxima and minima of this magnetism. It was then found that these points were not constant, but shifted their position as the plate was made to revolve in its own plane; or, in other words, that a plate which, in a given position, produced a certain deviation in a compass, no longer produced the same deviation after making an exact revolution in its own plane, although brought to rest, and every part of the apparatus restored precisely to its former place.

It appeared from this, that the revolution of the plate in its own plane had an influence on its power of deviating the needle independent of the partial magnetism of particular points. in it; and the justice of this idea was proved by giving it a rotation in an opposite direction, when the effect on its directive power was also reversed.

The change produced by rotation in the directive power of the plate was found to be a maximum when its plane was parallel to the line of dip on the magnetic axis, and at the same time as little inclined to the horizon as this condition would allow; but when the plane of the plate was parallel to the horizon, the effect was diminishred in the ratio of 5 to 1 , and when perpendicular to the horizon, and coincident with the magnetic meridian, was altogether destroyed. 
The author, having satisfied himself of the reality and constancy of this effect, in different plates, and of the necessity of referring it to a peculiar agency of the earth's magnetic power on the molecules of the plate, proceeded to ascertain the laws, and measure the quantities of the deviation due to rotation (so he terms it) in various positions; and detailed a great number of experiments, with their numerical results, arranged in the form of tables.

From these he deduced the following general law; viz. that the deviation due to rotation in a dipping needle "will always be such, that the sides of the equator of such dipping needle will deviate in a direction contrary to the directions in which the edge of the plate moves, that edge of the plate nearest to either edge of the equator producing the greatest effect."

The results of this law, it may be here observed, are in many cases coincident with those of the following : conceive the dipping needle orthographically projected on the plate. Then will the deviation due to rotation of the projected needle take place in a direction opposite to that of the rotation itself.

The author then proceeded to a theoretical investigation of the effect of a plate of soft iron, having within it two poles developed in given positions, and acting (in addition to the usual magnetic action of soft iron) on a needle of infinitely small dimensions, in the plane of the plate. He referred the whole ordinary action of the iron to its centre, and supposed that this is attractive on both poles of the needle; but the extraordinary action on that of the newly-developed poles he supposed to reside in them, and to be attractive or repulsive, according as they act on the poles of the needle of the same or opposite names with themselves. On this hypothesis, assuming symbols for the co-ordinates of the plate's centre, the distance separating the newly-developed poles in the plate, and the angle which the line joining them makes with the direction of the needle, \&c., he deduced (from the known laws of magnetism) formulæ, expressing the horizontal deviations of the needle:-first, on the supposition of a rotation in one direction; secondly, on that of a rotation in the opposite; and thirdly, in that of no rotation at all. From these, by comparing them with a few of the observations, he deduced numerical values for the constants of the formulæ, and then employed them to compute the deviations due to rotation in all the rest. He regarded the discrepancy between the calculated and observed results, as in few cases, larger than what he considered may be fairly attributed to error of observation; and that the theory above stated is at least a general representation of what passes in fact : admitting, however, that it does not give the exact posi- 
tion of the point where the deviation due to rotation vanishes, and suggesting partial magnetism in the iron plate used as one mode of accounting for the difference. At all events, by an examination of the case on the ordinary supposition of induced magnetism in the iron, he showed that a greater coincidence between theory and fact would not result from that hypothesis than from the one here employed.

He then proceeded to inquire into the degree of permanence of the polarity thus produced in iron by rotation; from which inquiry it appeared that (at least during 12 hours after the plate was brought to rest) the influence of a single rotation had scarcely suffered any diminution. It appeared also that the effect is so far from depending on the rapidity of the motion, that the plate can scarcely be made to revolve so slowly as that the whole effect shall not be produced.

Lastly, by a slight change in the formulæ, the results of computation, it is found, can be made to agree with observation to a degree of exactness as near as can be wished. This change consists in the omission of certain terms introduced by the theory, and the author regards it as very possible so to modify the theory as to get rid of them.

The author closed this communication with an appendix comparing the magnetic effects produced by slow and rapid rotation. The result of the comparison was, that the forces exerted on the needle during rapid rotation were always in the same direction as those derived from the slowest rotation, and which continue to act after the rotation has ceased, but were greater in intensity, and that the former effects were such as might have been looked for from a knowledge of the latter.

An Account of the Repetition of M. Arago's Experiments on the Magnetism developed during the Act of Rotation. By Charles Babbage, Esq. F.R S., and. J. F. Herschel, Esq. Sec. R.S.

The experiments of M. Arago having excited much interest, the authors of this communication were induced to erect an apparatus for their verification; and after a few trials, they succeded in causing a compass to deviate from the magnetic meridian, by setting in rotation under it plates of copper, zinc, lead, \&c.

To obtain more visible and regular effects, however, they found it necessary to reverse the experiment, by setting in rotation a powerful horse-shoe magnet, and suspending over it the various metals, and other substances to be examined, which were found to follow with various degrees of readiness the motion of the magnet. The substances in which they succeeded

Vol, 66. No. 328. Aug. 1825.

$\mathbf{N}$ 
in developing signs of magnetism were, copper, zinc, silver, tin, lead, antimony, mercury, gold, bismuth, and carbon in that peculiar metalloidal state in which it is precipitated from carburetted hydrogen in gas works. In the case of mercury, the rigorous absence of iron was secured. In other bodies, such as sulphuric acid, rosin, glass, and other non-conductors, or imperfect conductors of electricity, no positive evidence of magnetism was obtained.

The comparative intensities of action of these bodies were next numerically determined by two different methods, viz., by observing the deviation of the compass over revolving plates of great size cast to one pattern, and by the times of rotation of a neutralized system of magnets suspended over them; and it is curious that the two methols, though they assigned the sane urder to the remaining bodies, uniformly gave opposite results in the cases of zinc and copper, placing them constantly above or below each other according to the mode of observation employer.

Our authors next investigated the effect of solution of continuity on the various metals: in the course of which M. Arago's results of the diminution of effect by division of the metallic plates used were verified; and the further curious fact ascertained, that re-establishing the metallic contact with other metals restores the force, either wholly or in great measure; and that even when the metal used for soldering has, in itself, but a very feeble magnetic power, thus affording a power of magnifying weak degrees of magnetism. The law of diminution of the force by increase of distance was next investigated. It appears to follow no constant progression according to a fixed power of the distance, but to vary between the square and the cube.

The remainder of this paper was devoted to some able and elaborate reasoning on the facts detailed.-The authors conceive that they may be all explained without any new hypothesis in magnetism, by supposing simply that time is requisite both for the development and loss of magnetism; and that different metals differ in respect, not only of the time they require, but in the intensity of the force ultimately producible in them; and they apply this explanation not only to their own results, but to those obtained by $\mathrm{Mr}$. Barlow in his paper on the rotation of iron.

Experiments on the Magnetism produced by Rotation.

S. H. Christie, Esq., in a Letter to Mr. Herschei.

Mr. Christie, in this communication, gave an account of some experiments on the development of magnetism in copper 
by rotation. He corroborated by his own experience the results obtained by Mr. Herschel, in which a disc of copper was set in rotation by the rotation of one or more magnets beneath it, both in the case where poles of the same name were immediately below the disc, and when of a contrary name. The actions appeared equally intense in both cases; and from this circumstance, he concludes the magnetism thus communicated to the copper to be extremely transient. The experiment was varied by combining the revolving magnets differently, and the results were stated.

The next experiments of Mr. Christie were directed to the determination of the law according to which the force diminishes as the distance between the disc and magnets increases. It seems to follow from these experiments, that when a thick copper plate is made to revolve under a small magnet, the force tending to deviate the needle is directly as the velocity, and inversely as the fourth power of the distance; but that when magnets of considerable size are made to revolve under thin copper discs, the diminution follows more nearly the ratio of the inverse square of the distance, or between the square and the cube, though not in any constant ratio of an exact power.

The author then investigated the law of force when copper discs of different weights are set in rotation, which, for small distances, appear proportioned to the weights of the discs, but for smaller ones appear to vary in some higher ratio.

\section{Account of the Repetition of M. Arago's Experiments on the Magnetism developed during the Act of Rotation. By Messrs. Barlow and Marsh.}

The experiments by Mr. Barlow on the magnetism imparted by rotation, described in p. 93, were begun in Dec. 1824; and it was not until April 1825 that he was informed of M. Arago's rotative experiments on copper and other metals. "The latter were not known in England," Mr. Marsh states, "until M. GayLussac's visit to London at the time above stated." - I am not aware," he continues, "of the precise nature of these experiments; and shall, therefore, only endeavour to describe those which I have assisted Mr. Barlow in making, and which he founded on the description he had received: they may, therefore, be considered as the experiments of $M$. Arago repeated, and varied as different circumstances occurred to suggest new ideas. The account he had of M. Arago's experiment, was that, by placing a copper plate upon a vertical spindle, the plate being horizontal, and then placing just above it a light compass needle, but independent, of course, of the

$$
\text { N } 2 \text { plate; }
$$


plate; on causing the spindle and plate to revolve, the needle was considerably deflected, and more and more as the velocity was increased; so that, when the plate was put into rapid rotation, the needle also began, after a few vibrations, to revolve, and at length with considerable velocity.

" 1 . In order to repeat this experiment, I connected the wheel of my turning lathe with a vertical spindle, which I could make revolve forty-five times per second; and on this I placed $a$ thin copper plate, about six inches in diameter, and over this a needle about five inches long, shut up in a close box, about one inch, or rather less, above the plate. When putting the lathe in motion, I found it to deflect the needle about five points, the deflection being always in the same direction as the motion of the plate, but we could not cause it to revolve. The needle was, therefore, partly neutralized by a bar magnet, and the experiment repeated. We then very soon obtained a considerable rotatory motion in the needle; and, by using a larger and heavier plate, the same was produced afterwards without neutralizing the needle.

"2. Another experiment, which was mentioned as one of M. Arago's, and which I repeated, was, by interposing a plate of iron between the copper plate and the needle. In this case, no effect could be produced on the needle by the rotation of the copper plate, the iron clearly intercepting the action.

"3. The only other experiment that I am aware of as originating with M. Arago, at least that I repeated, was the rotation of a plate cut into radii like a star, which was said, as I understood, to produce no effect: this, however, was not the case in my experiments, -it certainly produced a less effect, but, I think, not less than might have been anticipated, from the quantity of copper thus taken away.

" 4 . I now tried a zinc plate instead of a copper plate, and the effect was nearly the same as before, but a little less.

" 5. An iron plate was now substituted, and the effect was considerably greater than with the copper plate.

" 6 . The copper plate was again replaced, and a brass needle placed in the box. Some motion was obtained, but it was very equivocal, so that I cannot venture to say that it was certainly due to the rotation.

66.7. A heavy horse-shoe magnet was now suspended by a line from the ceiling; and it was put in rotation by the revolution of the copper plate, a paper screen having been first interposed between them.

"8 8. One copper plate was suspended over another, but no motion was obtained; and the same took place when the copper plate was suspended over an iron one. 
" 9. A bar magnet, rather shorter than the diameter of the copper plate, was fixed horizontally to the upright spindle; and being made to revolve, the plate very soon acquired rotation. A paper screen was, in this, as in the preceding experiments, interposed between the plate and magnet.

"10. The plate was now applied immediately to the axis of the lathe, so as to cause it to revolve vertically, and the needle placed near to it; but no motion took place, till, by nearly neutralizing the needle, and bringing either of its poles directly to the plate, it then always deviated in the direction of the motion of the plate; whichever pole of the needle was directed to the former. The needle, of course, therefore, deviated different ways (all other things being the same), when it was above or below the axis; but in the direct horizontal line of the axis no motion in the needle took place.

"11. 'The above are the principal experiments that Iassisted in making by revolving the plate; but these having suggested to Mr. Barlow that all the results obtained might be explained, by supposing that there existed a slight magnetic power in copper, and in the various metals which had a tendency to draw the needle after the plate, or the latter after the former, he endeavoured to exhibit this by direct experiment, independent of revolution. With this view, he neutralized a needle very accurately; and then applying very near to its poles the end of a round brass ruler, the attraction of the latter was obvious, - it drew the needle several degrees,-then, withdrawing it, and catching the needle again in its returning vibration, it was drawn out some further degrees ; and, in a very short time, the deflection was converted into a revolution, which, by alternately presenting and withdrawing the needle, was at length rendered very rapid.

"6 12. The same result was obtained by two or three different pieces of brass; but there were other pieces, although of the same size and form, which had little or no effect.

"T The following experiment is due to Mr. Sturgeon, of Woolwich.

" 13. A thin copper plate or wheel, about five or six inches in diameter, was suspended very delicately on an axis, and then one side a little weighted, in order to give it a tendency to oscillate. The heavy point was now raised level with the axis, and the number of vibrations the plate made before it came to rest were counted. The same was again done, with this difference only, that the vibrations now took place between the poles of a horse-shoe magnet; and the number of them before the plate came to rest, was very little more than one half of what they were in the former instance. 
" This is the converse of M. Arago's experiments, in which he shows the effect of copper and other metallic rings, in diminishing the number of oscillations of a magnetic needle.

" 14. If, instead of a horse-shoe magnet, the contrary poles of two bar magnets be used, the effect is the same as before; but, if the poles of the same name, viz. both north or both south, be employed, then the effect is scarcely perceptible. This is an important result, as it shows that the effect is no due to any kind of resisting medium, as was supposed in the first instance."-Edin. Phil. Journ.

XIII. On the Conditions of Possibility, Arbitrary Functions, and Complete Solutions of Periodical Functional Equations. By John Herapath, Esq.

GOLUTIONS of functional equations have been considered to be of three kinds; particular, general, and complete. It has also been usual to consider the complete solution of any equation of the first order, as for instance of

$$
\mathrm{F}\left\{x, \psi x, \psi \alpha x, \psi \alpha^{2} x, \ldots \psi \alpha^{n-1} x\right\}=0
$$

to contain $n-1$ arbitrary functions; and with respect to the form of $\mathrm{F}$, it seems to have been tacitly admitted to be quite unlimited. Having been led by my inquiries to different conclusions, I purpose in the present paper to examine the conditions of possibility of (1), the limitations to the form of $F$, and to give a simple direct method of obtaining the complete solution. I regret only that my very confined limits will oblige me to be less explicit in my exemplifications than I could wish.

\section{Of the Conditions of Possibility.}

When any equation of the form of (1) is given, it may be put under the form of

$$
\psi x=f\left\{x, \psi \alpha x, \psi \alpha^{2} x, \ldots \psi \alpha^{n-1} x\right\}
$$

and there must simultaneously subsist the $n-1$ following equations,

$$
\begin{aligned}
& \psi \alpha x=f\left\{\alpha x, \psi \alpha^{2} x, \psi \alpha^{3} x, \ldots \psi x\right\} \\
& \psi \alpha^{2} x=f\left\{\alpha^{2} x, \psi \alpha^{3} x, \psi \alpha^{4} x, \ldots \psi \alpha x\right\}
\end{aligned}
$$

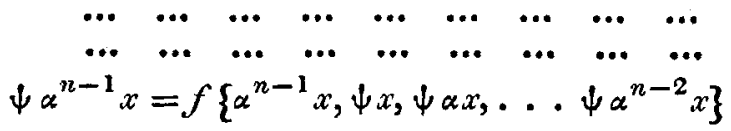

If now these $n-1$ right-hand functions be substituted for their values $\psi \alpha x, \psi a^{2} x, \ldots$ in (2), the resulting right-hand 